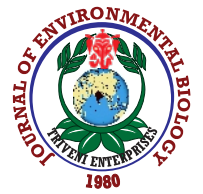

\title{
New cost-effective mediator enhanced enzymatic degradation of aniline blue
}

\author{
C. Lü1, X.F. Luo', X.J. Dong', J.Q. Peng ${ }^{1}$ and F.X. Cao ${ }^{1 *}$ \\ 'School of Life Science and Technology, Central South University of Forestry and Technology, Changsha-410004, China \\ ${ }^{2}$ School of Chemical and Biological Engineering, Hunan University of Science and Engineering, Yongzhou-425 000, China \\ *Corresponding Author Email : klhlv@126.com
}

\section{Abstract}

Aim: In this study, the effects of various agricultural wastes as low-cost, readily available alternatives to chemical mediators for the enzymatic degradation of aniline blue were investigated.

\begin{abstract}
Methodology: Enzymes for degrading aniline blue were obtained through solid-state fermentation (SSF) of Pleurotus eryngii. Agricultural waste including grape skin/seed and orange peel, rich in organic acids, were used instead of chemical reagents as mediators. The degradation products were subjected to liquid chromatography-mass spectrometry (LC-MS) analysis.
\end{abstract}

Results: The results show that the enzymes manganese peroxidase and versatile peroxidase were associated with aniline blue degradation. The organic acid-rich agricultural waste (grape seed/skin, orange peels) serving as substitutes for chemical reagents as mediators reduced the cost of the mediator by $99.8 \%$ at best, and the $2 \mathrm{hr}$ degradation rate of aniline blue was $97.4 \%$ and $95.8 \%$, respectively, increased by at least $4.3 \%$. The LC-MS analysis revealed that aniline blue was degraded into smaller compounds with significantly lower toxicity.

Interpretation: Overall, the enzyme-organic acid-

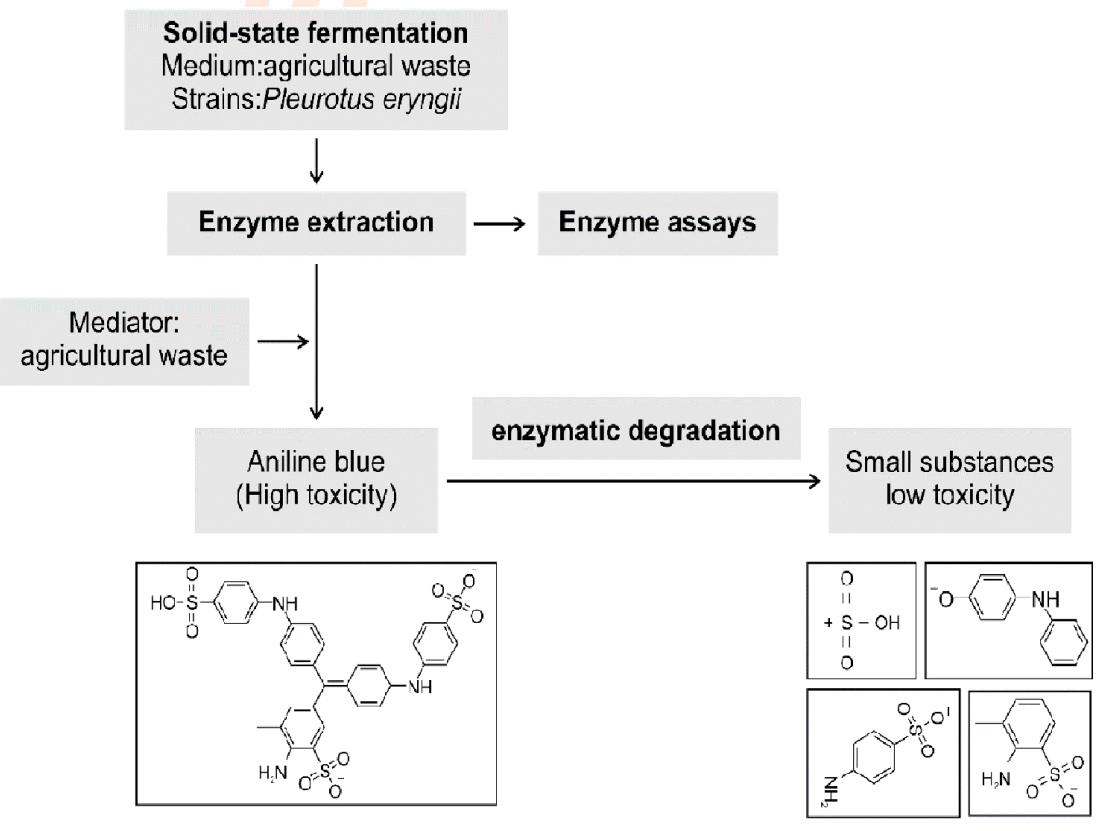
rich agricultural waste mediator (grape seed/skin) system effectively degraded aniline blue. This cheap and efficient method for aniline blue degradation can serve as an environmentally friendly technology to treat waste with waste.

Key words: Agricultural waste, Aniline blue, Enzymatic degradation, Organic acids, Pleurotus eryngii

How to cite : Lü, C., X.F. Luo, X.J. Dong, J.Q. Peng and F.X. Cao: New cost-effective mediator enhanced enzymatic degradation of aniline blue. J. Environ. Biol., 42, 99-105 (2021). 


\section{Introduction}

Aniline blue, an extensively used toxic dye, is a member of triphenylmethane dyes (Li et al., 2020). The trisulfonic acid group in aniline blue confers high polarity to the molecule, thereby deterring its degradation and increasing environmental toxicity ( $\mathrm{Li}$ et al., 2018). Aniline blue is degraded by chemical (Zhang et al., 2017), physical (Ayed et al., 2010), or biological methods (Anastasi et al., 2011). The biological method involves microbial degradation and biological enzymatic degradation (Zhang et al., 2017). The chemical and physical methods have disadvantages of high cost, low efficiency, and secondary contamination (Ayed et al., 2017; Holkar et al., 2014). Microbial degradation is reportedly unsuitable for aquatic environments, and the dye degradation potential of microorganisms in aquatic environments is low, probably because these microorganisms do not grow efficiently in aquatic environments (Li et al., 2018).

Previous studies have reported that prior upregulation of the required enzymes is the most efficient method for degrading dyes (Ma et al., 2016; Kittl et al., 2012). Microbial degradation of dyes primarily depends on enzymes related to lignin degradation, such as laccase, lignin peroxidase and manganese peroxidase (Grandes-Blanco et al., 2020; Chmelová and Ondrejovic, 2016; Jia et al., 2004; Wang et al., 2016). Pleurotus eryngii, a white-rot fungus, has a high potential to produce various ligninolytic enzymes capable of degrading lignin, cellulose and hemicelluloses. It is widely used to degrade persistent pollutants; therefore, $P$. eryngii is considered a model organism with extensive biotechnological applications (Palma et al., 2016). Studies have reported that solid-state fermentation of agricultural wastes for enzyme production by $P$. eryngii is a cost-effective, efficient, and sensible method for the degradation of such waste materials (Lu et al., 2019; Palma etal., 2016; Akpinar and Urek, 2014).

After enzyme acquisition, the selection of an appropriate mediator is a critical step in the enzymatic degradation of dye. Mediators play an important role by increasing the biodegradation efficiency of dyes in enzymatic degradation system. Mediators maintain a stable redox potential during the reaction and are converted into diffusible oxidants, thus overcoming steric hindrance and facilitating catalysis (Morales et al., 2012). Zhang et al. (2017) reported that the degradation rate of aniline blue was only $8.5 \%$ ( $8 \mathrm{hr}$ ) without mediators and that it increased to $89.6 \%$ with the use of 1-hydroxy benzotriazole as a mediator. Jia et al. (2004) reported that the dye degradation rate was $59.7 \%$ (24 hr) and that it increased to $70.1 \%$ with the use of veratryl alcohol as the mediator. However, mediators are expensive; hence, the cost associated with these degradation methods is high and is not conducive for large-scale use. In a previous study, a cost-effective enzyme-producing medium based on agricultural waste was reported (Lu et al., 2019). In line with this report, the aim of this study was to identify few potent agricultural waste products as low-cost, effective mediators (instead of expensive chemical reagents) and to elucidate the effect of enzyme-agricultural waste mediator system on aniline blue degradation.

\section{Materials and Methods}

Strains and agricultural waste: Pleurotus eryngii strain number 1 was provided by the Hunan Province Microbiology Institute, Changsha, China. The fungal culture was transferred to potato dextrose agar and incubated at $30^{\circ} \mathrm{C}$ for inoculum preparation. Wheat bran, banana peel, potato peel, garlic husk, orange peel, grape seed/skin, used tea leaves, and corn husk were purchased from a local market in Changsha, China, washed with distilled water and oven dried (Shanghai Precision Instrument, China), until an equilibrium moisture content of approximately $10 \%$ was acquired. The dried wheat bran and banana peel were crushed into 20-mesh particles and other materials were crushed into 100 mesh particles.

Enzyme preparation: The production of ligninolytic enzymes by P. eryngii was evaluated by solid-state fermentation (SSF) using 6 $\mathrm{g}$ of wheat bran, $300 \mathrm{mg}$ of banana peel, and $18 \mathrm{ml}$ of aniline blue solution (200 $\mathrm{mg} \mathrm{l}^{-1}$ ) (Sinopharm Chemical Reagent, Shanghai, China) or $18 \mathrm{ml}$ of distilled water as media. The media were sterilized and dispensed into $250 \mathrm{ml}$ Erlenmeyer flasks. Two agar plugs of mycelium ( $1 \mathrm{~cm}$ disks) were used as inoculum. The flasks were statically incubated at $28^{\circ} \mathrm{C}$ for 17 days. Samples for SSF were mixed with $60 \mathrm{ml}$ of distilled water and incubated in an oscillation incubator (Shanghai Precision Instrument) for $1 \mathrm{hr}$ with continuous stirring $(180 \mathrm{rpm})$ at $30^{\circ} \mathrm{C}$, and then separated via centrifugation at $4^{\circ} \mathrm{C}$ and $10,000 \times g$ for $10 \mathrm{~min}$ (high-speed refrigerated centrifuge; Sigma, Osterode am Harz, Germany). As described by Palma et al. (2016), the supernatant, a crude enzyme solution, was used to determine enzyme activity and degrade aniline blue. Lac activity was assessed following the method of Munoz et al. (1997), using ABTS (Songon Biotech, Shanghai, China) as the substrate in sodium succinate buffer (Sinopharm Chemical Reagent) at pH 4.0. One unit of Lac activity corresponded to the oxidation of $1 \mu \mathrm{mol}$ ABTS per minute.

$\mathrm{MnP}$ activity was estimated following the method of Wariishi et al. (1992) using $\mathrm{Mn}_{2} \mathrm{SO}_{4}$ (Sinopharm Chemical Reagent) as substrate in sodium malonate buffer (Sinopharm Chemical Reagent) at $\mathrm{pH}$ 4.5. One unit of $\mathrm{MnP}$ activity corresponded to the oxidation of $1 \mu \mathrm{mol} \mathrm{Mn}^{2+}$ per minute. VP activity was assessed following the method of Sarnthima et al. (2009), using DMP (Songon Biotech) as the substrate in sodium malonate buffer (Sinopharm Chemical Reagent) at pH 4.5. One unit of VP activity corresponded to the oxidation of $1 \mu \mathrm{mol}$ DMP per minute. LiP activity was assessed following the method of Tien and Kirk (1984) using VA (Songon Biotech) as substrate in tartaric acid buffer (Sinopharm Chemical Reagent) at pH 2.5.

One unit of LiP activity corresponded to the oxidation of 1 umol VAper minute.

Degradation of aniline blue: The $4 \mathrm{ml}$ degradation reaction system comprised $3.3 \mathrm{ml}$ of dye solution $\left(100 \mathrm{mg} \mathrm{l}^{-1}\right), 0.6 \mathrm{ml}$ of crude enzyme solution, $0.1 \mathrm{ml}$ of $\mathrm{H}_{2} \mathrm{O}_{2}$ solution ( $16 \mathrm{mM}$ ), $7 \mathrm{mg}$ of different types of pretreated agro-industrial waste or VA $(2 \mathrm{mM})$ as 
a mediator, and malonic acid buffer (2 mM) (Sinopharm Chemical Reagent). The blank set contained inactivated enzyme solution (at $100^{\circ} \mathrm{C}$ for $\left.5 \mathrm{~min}\right)$. The dye solutions before $(0 \mathrm{hr})$ and after (2 hr) degradation were analyzed using a UV-3100 spectrophotometer (Shanghai Precision Instrument) at the full visible and ultraviolet wavelengths $(200-700 \mathrm{~nm})$. The changes were compared in the absorption spectra.

The degradation rate was assessed following the method of Bandounas et al. (2013). To $5 \mathrm{ml}$ of each dye solution before ( 0 $\mathrm{hr}$ ) and after (the sample with the highest degradation rate in $2 \mathrm{hr}$ ) degradation, anhydrous ethanol precipitation (solution/ethanol, $1: 4, v / v)$ was added to eliminate proteins. After centrifugation $\left(4^{\circ} \mathrm{C}, 10,000 \times \mathrm{g}, 10 \mathrm{~min}\right)$, the supernatant was evaporated and re-dissolved in $1 \mathrm{ml}$ of methanol. The degradation products of aniline blue were detected by liquid chromatography-mass spectrometry (LC-MS) (Acquity ${ }^{\mathrm{TM}}$ ultra, Waters, USA), and the degradation products were inferred on the basis of changes in the substrate and product (Li et al., 2014).

Phytotoxicity analysis: Anhydrous ethanol precipitation (solution/ethanol $=1: 4, v / v$ ) of degraded aniline blue solution was carried out to eliminate proteins. After centrifugation $\left(4^{\circ} \mathrm{C}, 10,000\right.$ $\times \mathrm{g}, 10 \mathrm{~min}$ ), the degraded aniline blue solution was prepared through evaporation and drying. The phytotoxicity of aniline blue and its metabolites was studied using Chinese cabbage seeds, and the corresponding germination rate $(\%)$, root length $(\mathrm{cm})$, and seedling length $(\mathrm{cm})$ were measured as phytotoxicity indices. The Chinese cabbage seeds were cultured in a light incubator at $28^{\circ} \mathrm{C}$ (12-:12-hr light/dark cycle). Five milliliters of aniline blue solution, degraded aniline blue solution, and distilled water (as control) were added every day for 5 days and the aforementioned indices were determined (Baumgarten et al., 2004).

Statistical analysis: All experiments were performed independently in triplicate and the differences among treatment groups were analyzed by One-way analysis of variance (ANOVA). Results with $\mathrm{P}<0.05$ were considered statistically significant.

\section{Results and Discussion}

Dye biodegradation is primarily attributed to the catalytic activities of various related enzymes in organisms, such as Lac, LiP, MnP, VP and NADH-DCIP reductase and mediator supplementation effectively improves the catalytic activities of these enzymes (Akpinar and Urek, 2014; Du et al., 2011). The enzyme-mediator system is an effective method in dye biodegradation. Degradation of natural lignin and persistent compounds such as dye depends on enzymes and mediators (Geng et al., 2018). Different enzymes degrade different dyes (Li et al., 2020). Here, the enzymes potentially associated with aniline blue degradation are listed in Table 1.

The results showed that the activities of Lac and LiP in the fermentation broth were affected due to the presence/absence of aniline blue in the culture medium; however, the effect of inducing factor for aniline blue degradation on the activity of two enzymes was not significant $(P>0.05)$. It has been demonstrated that the degradation of aniline blue is catalyzed by Lac or LiP (Ma et al., 2016; Yuan et al., 2016); however, their degradation efficiency was not high, with only $8 \%$ in $8 \mathrm{hr}$ (Zhang et al., 2017). Here, the Lac and LiP activities were determined in the fermentation broth; however, no significant change was detected after addition of aniline blue. The VP activity significantly increased after the addition of aniline blue in the culture medium. Previous studies have shown that VP is especially important dye degrading agent owing to its complex structure, broad substrate specificity and catalytic versatility (Morales et al., 2012; Lu et al., 2019). The MnP activity significantly increased after the addition of aniline blue in the culture medium. Consequently, MnP was found to be associated with aniline blue degradation, concurrent with the

Table 1: Activity of enzymes associated with aniline blue degradation

\begin{tabular}{lcc}
\hline Enzyme & Culture medium without Aniline blue & Culture medium with Aniline blue \\
\hline $\mathrm{MnP}^{\mathrm{a}}$ & $14.68 \pm 2.42$ & $18.71 \pm 3.53^{*}$ \\
$\mathrm{LiP}^{\mathrm{a}}$ & $0.29 \pm 0.01$ & $0.26 \pm 0.03$ \\
$\mathrm{VP}^{\mathrm{a}}$ & $13.17 \pm 2.31$ & $16.62 \pm 2.48^{*}$ \\
$\mathrm{Lac}^{\mathrm{a}}$ & $6.45 \pm 0.97$ & $7.18 \pm 1.43$ \\
\hline
\end{tabular}

Data are mean of triplicate \pm S.E. Significantly different from control at ${ }^{*} P<0.05 .{ }^{a}$ Enzyme $U^{-1}$ of substrate.

Table 2: Phytotoxicity analysis

\begin{tabular}{llll}
\hline Parameter & Distilled water & Aniline blue solution & $\begin{array}{l}\text { Degraded Aniline } \\
\text { blue metabolite solution }\end{array}$ \\
\hline Germination percentage $(\%)$ & 89 & 34 & 61 \\
Seedling length $(\mathrm{cm})$ & $1.77 \pm 0.24$ & $0.54 \pm 0.08$ & $1.32 \pm 0.28$ \\
Root length $(\mathrm{cm})$ & $1.84 \pm 0.35$ & $0.21 \pm 0.06$ & $0.91 \pm 0.12$ \\
\hline
\end{tabular}




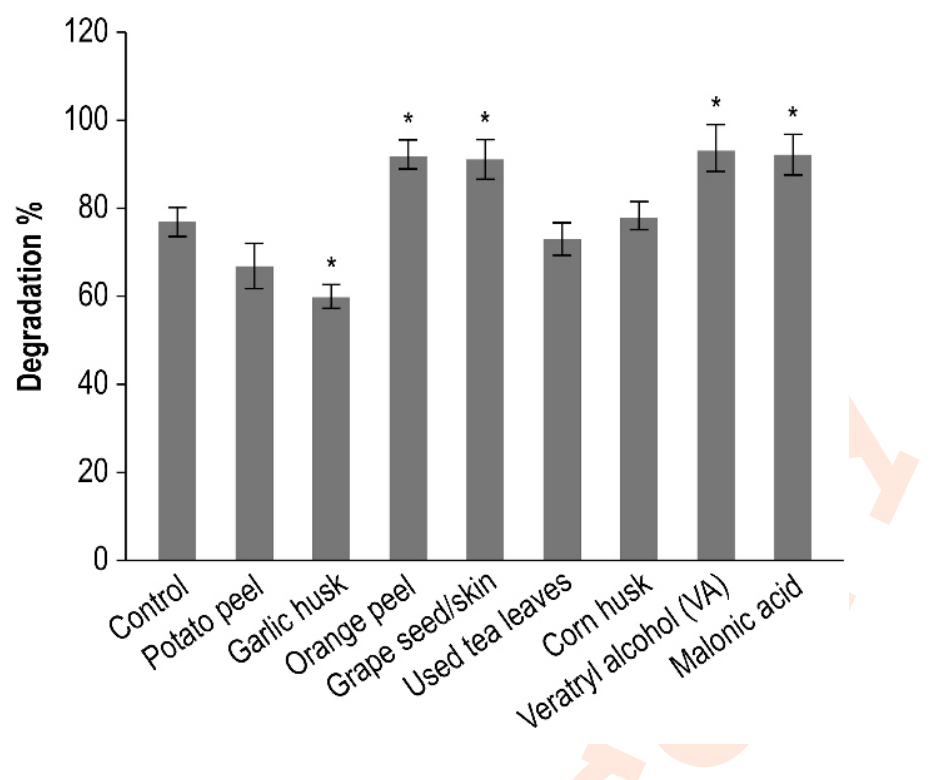

Fig. 1: Aniline blue degradation after the addition of different substances into the degradation system.

findings of previous studies on the enzymatic degradation of persistent pollutants (Anastasi et al., 2011; Tien and Kirk, 1984). Herein, the significantly increased activity of MnP and VP indicates that these enzymes are associated with aniline blue degradation.

Different mediators have different effects in the enzymatic degradation system. Moreover, mediator supplementation effectively improves the rate of enzymatic degradation as mediators can help overcome the spatial barrier between the active center of the enzyme and the substrate as well as the mediators can be transformed into small, diffusible oxidants (Bandounas et al., 2013). However, most commonly used mediators are expensive chemical reagents; hence, low-cost, readily available alternatives are urgently required (Palma et al., 2016). Accordingly, this study investigated the effect of various agricultural wastes as low-cost, readily available mediators to substitute for chemical reagents in enzymatic aniline blue degradation. As shown in Fig. 1, grape seed/skin and orange peel were used as mediators for enzymatic aniline blue degradation, and they were $4.3 \%$ and $2.7 \%$ more effective than VA and $7.2 \%$ and $5.4 \%$ more effective than malonic acid, respectively. These are commonly used mediators, probably because grape seed/skin and orange peel are rich in organic acids and are excellent metal-chelating agents, forming a stable complex with high redox potential (Aarjoo et al., 2017). The chelated metal ions serve as low-molecular-weight diffusible oxidants that overcome steric hindrance (Chhabra et al., 2009) and penetrate the interior for degradation (Devi et al., 2010; Morales et al., 2012). Simultaneously, grape seed/skin and orange peel are cheap and easy to obtain. These agricultural wastes did not significantly affect the degradation rate, but significantly reduced the cost of using mediators in the enzymatic degradation of dyes.
The cost of enzymatic aniline blue degradation using grape seed/skin as a mediator was $99.8 \%$ lower than that using VA, $1.3 \%$ lower than that using malonic acid, $38 \%$ lower than that using HBT and $97.2 \%$ lower than that using VA for Congo red degradation (Jia et al., 2004). The degradation rate decreased after the addition of potato skin as a mediator, probably owing to the alkaloids in potato skin, which inhibit proteases (Wang and Fu, 2015). The degradation rate also decreased when garlic husk was used as a mediator, likely due to the numerous organic sulfur compounds in garlic skin, which can inhibit calcium transport, concurrent with the findings of a previous study, which reported that calcium ions promote dye degradation (Lanzotti et al., 2006). The degradation rate decreased when tea leaves were added as a mediator, probably owing to the polyphenols in tea, which eliminate free radicals and reduce the substrate utilization rate ( $\mathrm{Li}$ et al., 2014). The comparison of degraded and undegraded dye solutions, by ultraviolet-visible spectrometry $(200-700 \mathrm{~nm})$ and LC-MS analysis revealed the obliteration of absorption peak $(600 \mathrm{~nm})$ after degradation, yielding a new absorption peak at 254 $\mathrm{nm}$; moreover, fragment ions at $\mathrm{m} / \mathrm{z} 248,184,80$ and 171 were detected upon secondary MS analysis. These results indicate that aniline blue was first hydrated, and then the product, i.e., aniline blue alcohol was formed. This was followed by continuous oxidative cleavage and then desulphurization, decarboxylation, and finally cleavage of $\mathrm{C}=\mathrm{N}$ bonds, yielding smaller substances (Fig. 2). In summary, using organic acid-rich agricultural waste (e.g., grape seed/skin) as the mediators, instead of chemical reagents, was efficient in degrading aniline blue in a short period. The degradation rate of aniline blue approached $97.4 \%$ in $2 \mathrm{hr}$, and the mediator cost was reduced by $99.8 \%$ at best. In addition, the degradation products were smaller compared to those reported in previous studies (Li et al., 2020; Zhang et al., 2017), which may suggest the degradation was more impactful using this method. 


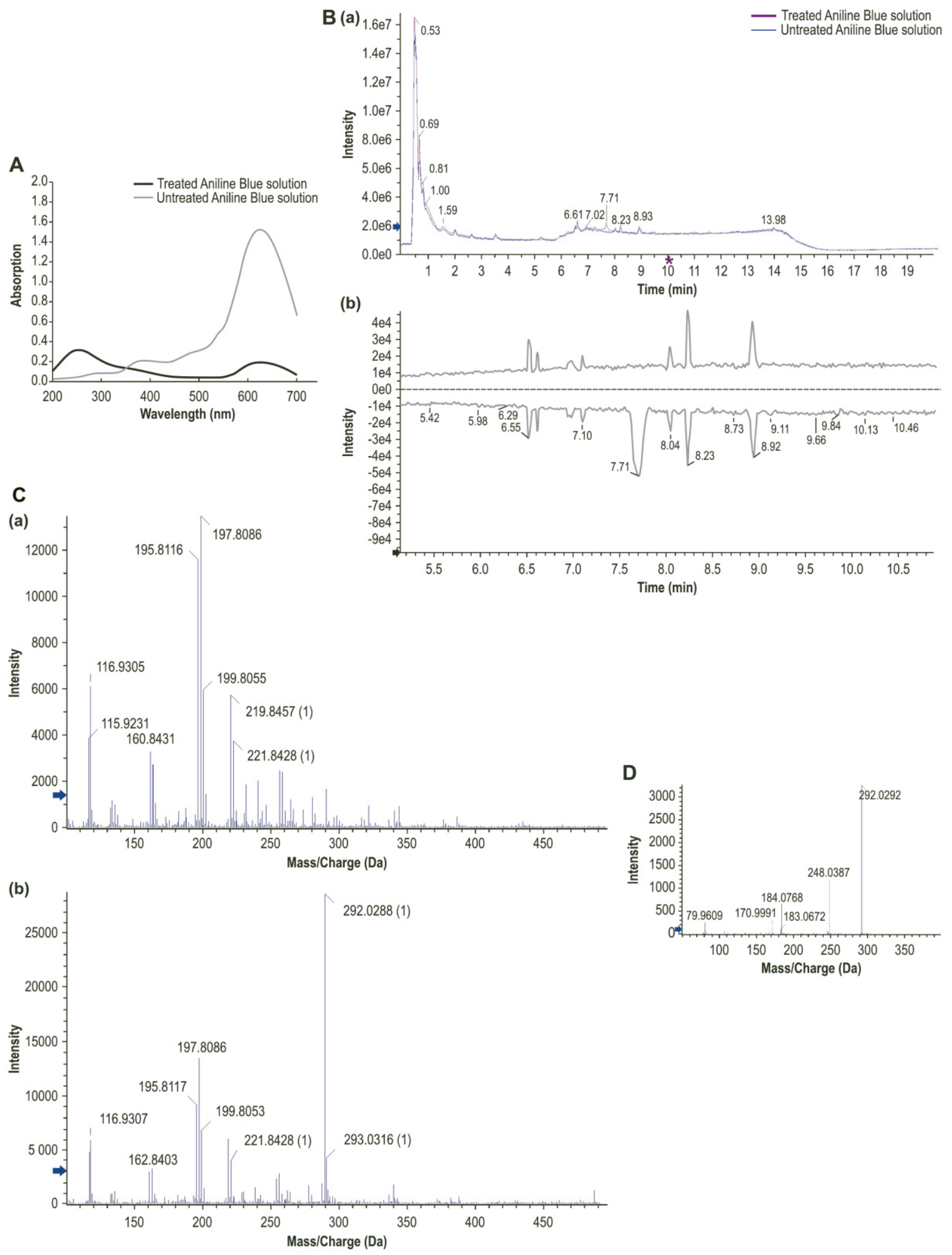

Fig. 2: (A) UV-visible spectra for treated and untreated Aniline Blue; (B) Total-ion chromatogram (a) and baes-peak ion chromatogram of undegraded (upward), degraded (downward) aniline blue (b); (C) Mass spectra for undegraded and degraded. (a) Mass spectrum for undegraded aniline blue (retention time, $7.71 \mathrm{~min}$ ), (b) Mass spectrum for degraded aniline blue (retention time, $7.71 \mathrm{~min}$ ) and (D) Secondary mass spectrometry of $\mathrm{m} / \mathrm{z}$ 292.0288 ions of degraded aniline blue. 
Aniline blue has high phytotoxicity, high residue level, and other toxic and adverse effects (Lu et al., 2019), probably owing to the lipophilicity and binding to the cell membrane (Ayed et al., 2017). The phytotoxicity of aniline blue and its degraded metabolites was assessed in this study using Chinese cabbage seeds, and their corresponding germination percentage, root length, and seedling length were measured and used as phytotoxicity indices. Compared with distilled water, the degraded aniline blue solution affected the germination rate, root length, and seedling growth of Chinese cabbage; however, the effect was less than that of aniline blue solution.

The degradation results suggest that the phytotoxicity of aniline blue may have been decreased by its degradation into small-molecule substances, enhanced metabolite hydrophilicity, and weakened binding to membrane lipids. In this study, the use of organic acid-rich agricultural waste as a mediator for enzymatic degradation of aniline blue were demonstrated to be a cost-effective, efficient and environmentally friendly method. The results of this study concluded that the enzyme-organic acid-rich agricultural waste mediator system provide novel insights into the degradation of dyes.

\section{Acknowledgment}

The authors are grateful for the financial support provided by the key project of Yibin City, China (No. 2017SF002).

\section{Add-on Information}

Authors' contribution: F.X. Cao: Propose ideas, design proposal, revise manuscript; C. Lü: Participate in experimental design, analyze data, write manuscript; X.F. Luo: Revise manuscript; X.J. Dong: Participate in experimental design, analyze data; J.Q. Peng: Guide experiments, Revise manuscript

Research content: The research contents is original and has not been published elsewhere

Ethical approval: NotApplicable.

Conflict of interest: The author declares that there is no conflict of interest.

\section{Data from other sources: NotApplicable}

Consent to publish: All authors agree to publish the paper in Journal of Environmental Biology.

\section{References}

Aarjoo, S., G. Vijaya, K. Mussarat, B. Sanjeev, G. Naveen, C. Neena and $S$. Prince: Flavonoid-rich agro-industrial residues for enhanced bacterial laccase production by submerged and solid-state fermentation. 3 Biotech, 7, 200 (2017).

Akpinar, M. and R.O. Urek: Extracellular ligninolytic enzymes production by Pleurotus eryngii on agroindustrial waste. Prep. Biochem. Biotechnol., 44, 772-781 (2014).

Anastasi, A., B. Parato, F. Spina, V. Tigini, V. Prigione and G.C. Varese: Decolorization and detoxification in the fungal treatment of textile wastewaters from dyeing processes. New Biotechnol., 29, 38-45 (2011).

Ayed, L., K. Bakir, H.B. Mansour, S. Hammami, A. Cheref and A. Bakhrouf: In-vitro mutagenicity, NMR metabolite characterization of azo and triphenylmethanes dyes by adherents bacteria and the role of the "CNA" adhesion gene in activated sludge. Microb. Pathog., 103, 29-39 (2017).

Ayed, L., K. Chaieb, A. Cheref and A. Bakhrouf: Biodegradation and decolorization of triphenylmethane dyes by Staphylococcus epidermidis. Desalination, 260, 137-146 (2010).

Chhabra, M., S. Mishra and T.R. Sreekrishnan: Laccase/mediator assisted degradation of triarylmethane dyes in a continuous membrane reactor. J. Biotechnol., 143, 69-78 (2009).

Chmelová, D. and M. Ondrejovic: Purification and characterization of extracellular laccase produced by Ceriporiopsis subvermispora and decolorization of triphenylmethane dyes. J. Basic Microbiol., 56, 1173-1182 (2016).

Devi, L.G., N. Kottam, B.N. Murthy and S.G. Kumar: Enhanced photocatalytic activity of transition metal ions $\mathrm{Mn}^{2+}, \mathrm{Nl}^{2+}$ and $\mathrm{Zn}^{2+}$ doped polycrystalline titania for the degradation of Aniline Blue under UV/solar light. J. Mol. Catal. A:Chem., 328, 44-52 (2010).

Du, L.N., S. Wang, G. Li, B. Wang, X.M. Jia, Y.H. Zhao and Y.L. Chen: Biodegradation of malachite green by Pseudomonas sp. strain DY1 under aerobic conditions: Characteristics, degradation products, enzyme analysis and phytotoxicity. Ecotoxicology, 20, 438-446 (2011).

Geng, W., R.A. Venditti, J.J. Pawlak and H. Chang: Effect of delignification on hemicellulose extraction from switchgrass, poplar and pine and its effect on enzymatic convertibility of cellulose-rich residues. BioResources, 13, 4946-4963 (2018).

Grandes-Blanco, A.I., A.L. Antonio-Flores, R. Garcia-Barrientos, S. Tlecuitt-Beristain, G. Diaz-Godinez and L. Sanchez-Minutti: Agrofood waste employed to design and develop culture media for fungal growth. J. Environ. Biol., 41, 195-201 (2020).

Holkar, C.R., A.B. Pandit and D.V. Pinjari: Kinetics of biological decolorisation of anthraquinone based reactive blue 19 using an isolated strain of Enterobacter fusiformis F NCIM 5545. Bioresour. Technol., 173, 342-351 (2014).

Jia, R., B.K. Tang, X.B. Zhang and Y.M. He: Effects of veratryl alcohol and Tween80 on ligninase production and its roles in decolorization of azo by white-rot basidiomycete PM2. Chin. J. Biotechnol., 20, 302305 (2004).

Kittl, R., K. Mueangtoom, C. Gonaus, S.T. Khazaneh, C. Sygmund, D. Haltrich and R. Ludwig: A chloride tolerant laccase from the plant pathogen ascomycete Botrytis aclada expressed at high levels in Pichia pastoris. J. Biotechnol., 157, 304-314 (2012).

Lanzotti, V.: The analysis of onion and garlic. J. Chromatogr. A., 1112, 3$22(2006)$.

Li, F., N. Liang and J.Z. Sun: Degradation characteristics of a novel aniline blue-discoloring bacterial strain MP-13. Microbiology China, 47, 43-53 (2020)

Li, G., L. Peng, Z. Ding, Y. Liu, Z. Gu, L. Zhang and G. Shi: Decolorization and biodegradation of triphenylmethane dyes by a novel Rhodococcus qingshengii JB301 isolated from sawdust. Ann. Microbiol., 64, 1575-1586 (2014).

Li, H., R. Zhang, L. Tang, J. Zhang and Z. Mao: Evaluation of Bacillus sp. MZS10 for decolorizing Azure B dye and its decolorization mechanism. J. Environ. Sci., 26, 1125-1134 (2014). 
Li, J., X. Chen, J. Deng, Y. Wu, L. Liu, Y. Tu and Y. Zhou: Extraction and antioxidant activity in-vitro of okra flavonoids. Food Sci., 35, 121125 (2014).

Li, W., C. Li, Y. Xu, Q. Wang, X. Ma, S. Chen, J. Yu and Y. Yang: Isolation of a microorganic strain for the high volume degradation of aniline blue and its application in natural sewage treatment. Comput. Mol. Biosci., 8, 149-165 (2018).

Lu, C., X. Luo, X. Dong, J. Peng and F. Cao: Enhanced versatile peroxidase production by Pleurotus eryngii using saccharide- and fatty acid-rich agro-industrial waste in solid-state fermentation. $J$. Biobased Mater. Bioene., 13, 134-139 (2019).

Ma, Y.H., L.J. Li, M.H. Lu and J.C. He: Isolation of aniline blue-discoloring actinomyces and its decolorization characteristics. Acta Sci. Circum., 36, 4361-4366 (2016).

Morales, M., M.J. Mate, A. Romero, M.J. Martínez, A.T. Martínez and F.J. Ruiz-Dueñas: Two oxidation sites for low redox potential substrates: A directed mutagenesis, kinetic and crystallographic study on Pleurotus eryngii versatile peroxidase. J. Biol. Chem., 287, 41053-41067 (2012).

Palma, C., L. Lloret, L. Sepúlveda and E. Contreras: Production of versatile peroxidase from Pleurotus eryngii by solid-state fermentation using agricultural residues and evaluation of its catalytic properties. Prep. Biochem. Biotechnol., 46, 200-207 (2016).

Sarnthima, R., S. Khammuang and J. Svasti: Extracellular ligninolytic enzymes by Lentinus polychrous Lév. under solid-state fermentation of potential agro-industrial wastes and their effectiveness in decolorization of synthetic dyes. Biotechnol. Bioproc. E., 14, 513-522 (2009).

Tien, M. and T.K. Kirk: Lignin-degrading enzyme from Phanerochaete chrysosporium: Purification, characterization and catalytic properties of a unique $\mathrm{H}_{2} \mathrm{O}_{2}$ requiring oxygenase. Proc. Natl. Acad. Sci. U.S.A., 81, 2280-2284 (1984).

Wang, C.C. and D.Q. Fu: The research progress of alkaloids in solanaceous crops. Chin. Biotechnol., 35, 99-104 (2015)

Wang, F., M. Ai, G. Yang, J. Chen, X. Chen and F. Huang: Influence of carbon source on the production of extracellular ligninolytic enzymes by Phanerochaete chrysosporium. BioResources, 11, 5676-5686 (2016).

Wariishi, H., K. Valli and M.H. Gold: Manganese (II) oxidation by manganese peroxidase from the basidiomycete Phanerochaete chrysosporium. J. Biol. Chem., 267, 23688-23695(1992).

Yuan, X., G. Tian, Y. Zhao, L. Zhao, H. Wang and T.B. Ng: Degradation of dyes using crude extract and a thermostable and $\mathrm{pH}$-stable laccase isolated from Pleurotus nebrodensis. Biosci. Rep., 36, e00365 (2016).

Zhang, R.J., H.X. Li, J.H. Zhang and Z.G. Mao: On the technical condition and the mechanism of the aniline blue decolorization under the impact of the laccase/HOBT mediator system. J. Hlth. Saf. Environ., 15, 233-236 (2017). 